\title{
Stock market crashes are outliers
}

\author{
Anders Johansen ${ }^{1}$ and Didier Sornette ${ }^{2,3}$ \\ 1 CATS, Niels Bohr Institute, Blegdamsvej 17, DK-2100, Denmark \\ 2 Department of Earth and Space Science and Institute of Geophysics and \\ Planetary Physics University of California, Los Angeles, California 90095 \\ ${ }^{3}$ Laboratoire de Physique de la Matière Condensée, CNRS UMR6622 \\ Université de Nice-Sophia Antipolis, B.P. 71, Parc Valrose, 06108 Nice Cedex 2
}

\begin{abstract}
We call attention against what seems to be a widely held misconception according to which large crashes are the largest events of distributions of price variations with fat tails. We demonstrate on the Dow Jones Industrial Average that with high probability the three largest crashes in this century are outliers. This result supports the suggestion that large crashes result from specific amplification processes that might lead to observable pre-cursory signatures.
\end{abstract}

PACS numbers: $01.75+\mathrm{m} ; 02.50+\mathrm{s} ; 89.90+\mathrm{n}$ 
Stock markets can exhibit very large motions, such as rallies and crashes. These are the most extreme deviations from the ingrained Gaussian description that was first shaken by Mandelbrot (see [1] and references therein) when he proposed to use Lévy distributions. Lévy distributions are characterised by a fat tail decaying as a power law with index between 0 and 2. Recently, physicists have characterised more precisely the distribution of market price variations [2-4] and found that a power law truncated by an exponential provides a reasonable fit at short time scales (less than one day), while at larger time scales the distributions cross over progressively to the Gaussian distribution which becomes approximately correct for monthly and larger scale price variations. Alternative representations exist [5] corresponding to different models inspired from an analogy with turbulence. These two classes of descriptions can only be distinguished using higher order statistics [6].

This has led naturally to the idea that the stock market could exhibit selforganising behaviour [7-10] where large motions can occur relatively often in contrast to what is expected within the Gaussian description.

The purpose of this short note is to point out the shortcomings of this concept when applied to the very largest crashes. We would like to stress the danger of amalgamating the existence of large crashes with the existence of a fat tail in the distribution of stock market prices. Our message is that the largest crashes of this century are outliers.

This is suggested by figure 1, which shows the number of times a given level of draw down has occurred in the Dow Jones Industrial Average in the period 19001994. A draw down is defined as the cumulative loss from the last local maximum to the next local minimum. The number of draw downs DD smaller than $\approx 15 \%$ is well fitted by an exponential law

$$
N(\mathrm{DD})=N_{0} e^{-\mathrm{DD} / \mathrm{DD}_{c}}, \quad \text { with } D D_{c} \approx 1.8 \% .
$$

This exponential fall-off is compatible with the previous results [2-6] because the time scales is already in the cross-over regime converging to the Gaussian distribution. The three largest events seem to be outside the range of the exponential fit. These events are in chronological order: World War 1, Wall Street 1929.8 and Wall Street 1987.8. The largest is the crash of October 1987, then World War 1 and Wall Street 1929.8. 
To forestall any criticism related to the influence of the binning can have on the appearance of such a fit, we have re-binned distribution of draw downs using a 4 times larger bin, see figure 2. We still see an exponential distribution with approximately the same decay constant $\left(\mathrm{DD}_{c} \approx 2.2 \%\right)$ for all but the 3 largest crashes. Note that the decrease in the distance between the crash of 1929 and the exponential fit is partly due to the fact that the larger binning attributes a decrease of $22.0 \%$ to the crash and not the correct value of $23.6 \%$. Let us also mention that the draw down have all approximately the same duration: the average duration is 3.3 days to be compared with 3.2 for the 6 crashes larger than $15 \%$.

To quantify how much these three events deviates from (11), we can calculate what would be, according to (1), the typical return time of a draw down of amplitude equal to or larger than the second largest of $28.8 \%$. Expression (1) gives the number of draw down equal to or larger than DD. Then $N_{0}$ is simply the total number of drawdown larger than $1 \%$ in a century. The fit yields $N_{0}=2360$, which is not too far from the exact number 2789 of drawn downs larger than 1\%. Taking the largest decay constant $D D_{c}$ of the two fits to be conservative, expression (1) predicts the number of drawn downs equal to or larger than $28.8 \%$ per century to be $\approx 0.006$. The return time of draw down equal to or larger than $28.8 \%$ would then be the number of centuries $n$ such that $0.006 n \sim 1$. This yields $n \sim 160$ centuries. Taking the other value $D D_{c}=1.8 \%$ yields a return time of 3000 centuries. In contrast, the market has sustained two such events in less than a century.

As an additional test, we have used a more sophisticated null-hypothesis than that of an exponential and generated 10.000 surrogate data sets corresponding to approximately one million years using a $\operatorname{GARCH}(1,1)$ model estimated from the true index with a t-student distribution with four degrees of freedom [11]. Among these 10.000 surrogate data sets only two had 3 draw downs above $22 \%$ and none had 4 . However, 3 of these 6 "crashes" showed a rather abnormal behaviour in the sense that they were preceded by a draw up of comparable size as the "crash" (for real crashes, the reverse is often seen, i.e., large crashes are typically followed by large draw ups). This means that in a million years of "Garch-trading", with a reset every century, never did 3 crashes occur. Furthermore, none were preceded by log-periodic 
signatures as found for the 1929 and 1987 crashes [12, 14, 13].

Figure 3 shows the number of times a given level of draw up has occurred in the Dow Jones Industrial Average of the same period. Here, a power law

$$
N(\mathrm{UD})=\left(\frac{\mathrm{UD}^{(+)}}{\mathrm{UD}}\right)^{1+\mu}, \text { with } \mu \approx 2.0
$$

accounts reasonably well for the data except for the very smallest events. There are no outliers for the draw ups and the exponent is not unreasonably far from the value around 1.5 that is found at smaller time scales. However, the difference is significant and could be interpreted as an effective power law characterising the cross-over from the truncated Lévy law at short time scales to the Gaussian distribution at long time scales.

Crashes have recently been modelled as special critical crises [11-17]. The underlying hypothesis is that stock market crashes are caused by the slow buildup of powerful subterranean forces that come together in one critical instant. The use of the word "critical" is not purely literary here: in mathematical terms, complex dynamical systems such as the stock market can go through so-called "critical" points, defined as the explosion to infinity of a normally well-behaved quantity.

Both crashes and the price variations with their fat tail distributions and anomalous correlations are thus proposed to be the result of endogenous self-organising process, however they are not the same phenomenon. There is rather a coexistence of self-organisation and criticality. Here, self-organisation refers to the globally stationary state of the market in normal times with fat tail distributions. The criticality here describes the special times when a great crash occurs which has been documented to be preceded by a rising susceptibility and pre-cursory signals in a way similar to a critical instability. Such a coexistence between self-organisation and criticality has been recently demonstrated in a hierarchical model of earthquakes [19], in which a coexistence of self-organisation of the crust at large time scales and a critical nature of large earthquakes were found. The critical nature of the large cascades emerges from the interplay between the long-range correlations of the self-organised state and the hierarchical structure of interactions: a given level of the hierarchical rupture is like a critical point to all the lower levels, albeit with a finite size. The finite size effects are thus intrinsic to the process. 
The fact that large crashes are outliers implies that they are probably triggered by additional amplifying factors. An important consequence is that specific signatures of their presence could exist, as proposed in [11-17], similar to precursors before instabilities. This is in contrast to the almost total absence of precursors before a large avalanche which is NOT an outlier in a self-organised state [20]. 


\section{References}

[1] B.B. Mandelbrot, Fractals and scaling in finance: discontinuity, concentration, risk (New York, Springer, 1997).

[2] R.N. Mantegna and H.E. Stanley, Nature 376, 46-49 (1995); Nature 383, 587-588 (1996).

[3] J.-P. Bouchaud, D. Sornette and M. Potter, Option pricing in the presence of extreme fluctuations, in Mathematics of Derivative Securities, edited by MA.H. Dempster and S.R. Pliska, Cambridge University Press 1997, pp. 112-125; Arneodo, A., J.-P. Bouchaud, R. Cont, J.-F. Muzy, M. Potter and D. Sornette, 1996. Comment on "Turbulent cascades in foreign exchange markets", (cond-mat/9607120)

[4] R. Cont, Scaling and correlation in financial data, Eur.Phys.J.B., in press (condmat/9705075).

[5] Ghashghaie S., Breymann, W., Peinke, J., Talkner, P. and Dodge, Y., Nature 381, 767-770 (1996).

[6] A. Arnéodo, J.-F. Muzy and D. Sornette, Causal cascade in the stock market from the "infrared" to the "ultraviolet", cond-mat/9708012)

[7] Takayasu, H., H. Miura, T. Hirabayashi and K. Hamada, Physica A 184, 127-134 (1992).

[8] Levy, M., H. Levy and S. Solomon, J. Phys. I France 5, 1087-1107 (1995).

[9] Bak, P., K. Chen, J.A. Scheinkman and M. Woodford, Ricerche Economiche 47, 3-30 (1993); Bak, P., M. Paczuski and M. Shubik, Physica A 246, 430 (1997).

[10] G. Caldarelli, M. Marsili, Y.-C. Zhang, A Prototype Model of Stock Exchange, Europhys. Lett. in press, cond-mat/9709118).

[11] T. Bollerslev, J. Time Series Analysis 9, 121 (1988); We use the t-student distribution to account for the fat tail in the distribution of price variations.

[12] Sornette, D., A. Johansen and J.-P. Bouchaud, J.Phys.I France 6, 167-175 (1996). 
[13] Feigenbaum, J.A., and P.G.O. Freund, Int. J. Mod. Phys. 10, 3737-3745 (1996).

[14] D. Sornette and A. Johansen, Physica A 245, 411 (1997)

[15] J. A. Feigenbaum, P. G.O. Freund, Discrete Scale Invariance and the "Second Black Monday", cond-mat/9710324

[16] N. Vandewalle, Ph. Boveroux, A. Minguet and M. Ausloos, The krach of October 1987 seen as a phase transition: amplitudee and universality, preprint 97

[17] S. Gluzman and V. I. Yukalov, Renormalization Group Analysis of October Market Crashes, cond-mat/9710336

[18] A. Johansen, O. Ledoit and D. Sornette, Crashes as Critical Points, presented at the Annual Meeting of THE WESTERN FINANCE ASSOCIATION, June 17-20, 1998, Monterey, California, http://www.gsm.cornell.edu/wfa

[19] Y. Huang, H. Saleur, C. G. Sammis, D. Sornette, Precursors, aftershocks, criticality and self-organized criticality, Europhysics Letters, in press (http://xxx.lanl.gov/abs/cond-mat/9612065)

[20] I. Sammis and J.M. Carlson, Phys. Rev.Lett. 74, 4357-4357 (1995). 
Figure 1: Number of times a given level of draw down has been observed in this century in the Dow Jones Average. The bin-size is 1\%. A threshold of $1 \%$ has been applied. The fit is equation (1) with $N_{0} \approx 2360$ and $\mathrm{DD}_{c} \approx 0.018$.

Figure 2: Number of times a given level of draw down has been observed in this century in the Dow Jones Average. The bin-size is $4 \%$. A threshold of $1 \%$ has been applied. The fit is equation (11) with $N_{0} \approx 3358$ and $\mathrm{DD}_{c} \approx 0.022$.

Figure 3: Number of times a given level of draw up has been observed in this century in the Dow Jones Average. A threshold of $1 \%$ has been applied. The fit is equation (2) with $\mu \approx 2.0$. 


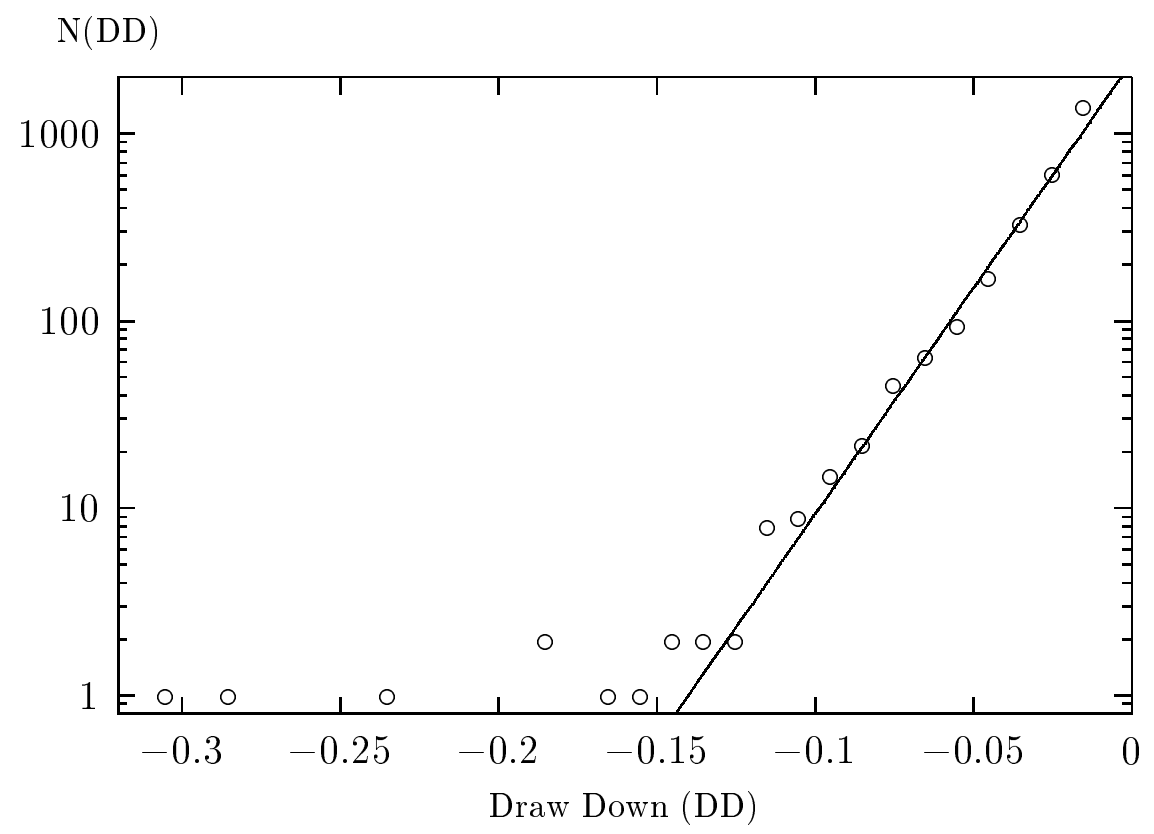









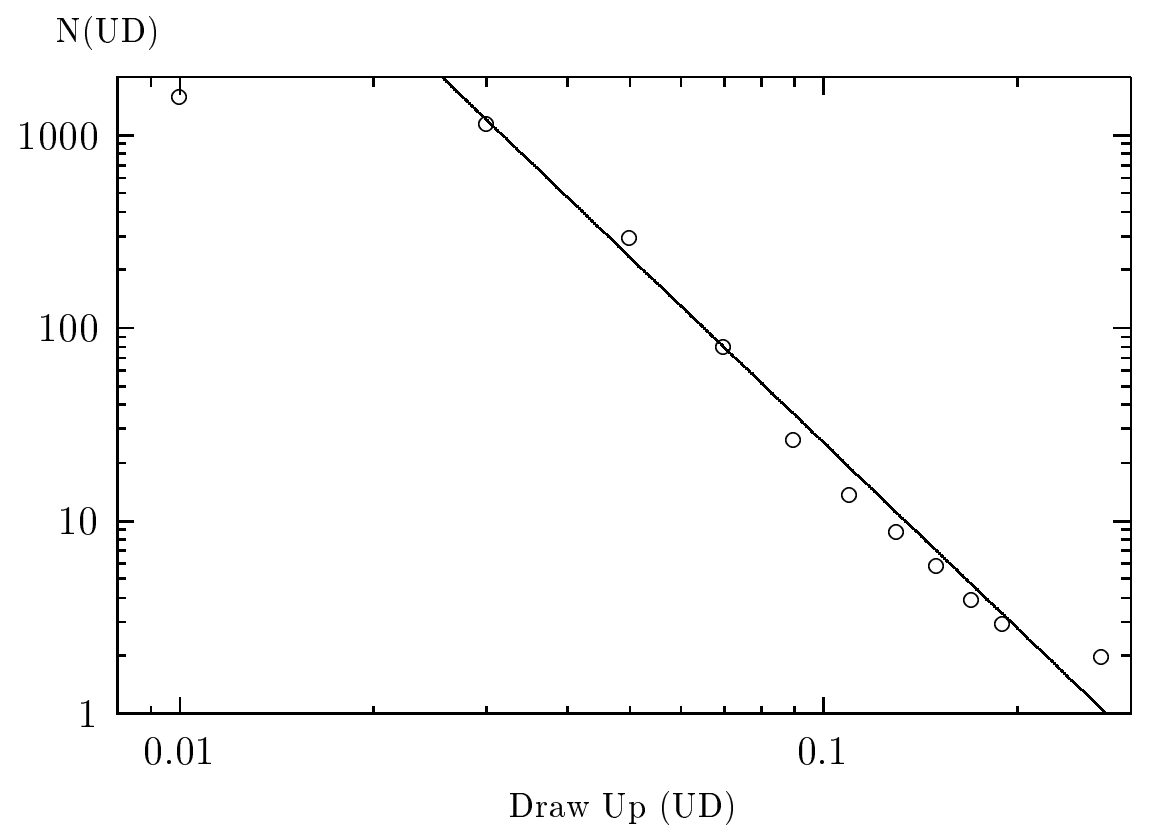

